\title{
Photoinduced Atomic Force Spectroscopy and Imaging of 2D Materials
}

\author{
T. U. Tumkur* ${ }^{\dagger}$, M. A. Hurier ${ }^{\dagger}$, M. B. Pichois, M. Vomir, B. Donnio, \\ J.L. Gallani, M.V. Rastei* \\ ${ }^{1}$ Institut de Physique et Chimie des Matériaux de Strasbourg, CNRS, Université de \\ Strasbourg, F-67034 Strasbourg, France \\ ${ }^{\dagger}$ Equal author contribution \\ * email : tumkur@ipcms.unistra.fr, $\underline{\text { rastei@ipcms.unistra.fr }}$
}

\begin{abstract}
We report on the near-field imaging of atomically thin layers of two-dimensional (2D) materials using photoinduced force mapping. This is accomplished by modifying a traditional atomic force microscopy setup to detect optical forces between a nanoscale tip and photoexcited sample. Our setup facilitates the imaging of few-layer flakes of $\mathrm{MoS}_{2}$ or $\mathrm{WS}_{2}$ and acquire optical force spectra, both in air and vacuum. The evaluated force spectra in both samples, exhibit the characteristic excitonic resonance peaks that are most typically observed in far-field absorption spectroscopy. We also show that nanoscale defect sites and flake edges can be distinguished from the crystalline flakes with high spectral resolution. Our results pave the way towards gaining a wholesome understanding of optical interactions and structure-property correlations in 2D materials and their heterostructures.
\end{abstract}

Two-dimensional (2D) sheets of atomically thin Van der Waals-bonded crystals have recently opened up new avenues for studying light-matter interactions at the nanoscale. A wide array of candidates including graphene and a palette of transition metal dichalcagonides (TMDs), have fueled the tremendous surge of scientific interest in 2D materials, which demonstrate remarkable optoelectronic 
and photonic properties. ${ }^{1-3}$ In the context of light-matter interactions, atomically thin layers of TMDs have a direct bandgap in the visible and near-infrared regions of the optical spectrum. ${ }^{2-5}$ Consequently, they have been shown to demonstrate extraordinary photoluminescence (PL), ${ }^{6,7}$ along with a host of other phenomena such as strong excitonic effects, ${ }^{8,9}$ large binding energies ${ }^{10,11}$ and photocatalytic activity. ${ }^{12,13}$ In combination with other photonic materials such as plasmonic structures or cavities, TMDs have been utilized to achieve strong light-matter coupling at room temperatures, ${ }^{14,15}$ hot-electron induced phase transition, ${ }^{16}$ ultrahigh Raman and Purcell enhancements, ${ }^{17-19}$ and low-threshold lasing. ${ }^{20,21}$

These fascinating properties of TMDs and 2D materials in general, depend strongly on fabrication conditions and on the presence of defects, impurities and grain boundaries. ${ }^{22-24}$ Moreover, defects in TMDs, have been shown to exhibit remarkable electronic properties ${ }^{25}$ and optical effects. ${ }^{26,27}$ As a result, understanding the correlation between the topological nature of atomically thin sheets and the subsequent evolution of optical properties is immensely important for their use in realistic applications and for the study of fundamental physical phenomena.

Several far- and near-field techniques have been employed to study and characterize the optical properties of 2D materials in the context of defect imaging. In particular, Raman and PL spectroscopies are routinely employed via optical microscopy techniques that are diffraction-limited. ${ }^{28}$ Tip-based imaging techniques such as scanning near-field optical microscopy (SNOM) and other hybrid tipenhanced techniques have employed PL and Raman signatures to identify nanoscale defects in 2D structures at relatively higher nanoscale resolutions. ${ }^{29-31}$ However, while it is typically assumed that absorption is homogeneous across several microns in PL-based studies of 2D structures, it has been shown that the presence of defects and structural disorders in atomic crystals lead to site-dependent absorption and PL responses. Moreover, PL-based characterization techniques are most useful only in 
the limit of single monolayers, as there exist a direct-to-indirect bandgap transition with increased thicknesses. ${ }^{2,4}$ Thus, a few studies have recently explored the possibility of characterization and defect mapping of 2D structures using absorption-based studies via SNOM, ${ }^{32}$ hyperspectral ${ }^{33}$ and confocal microscopy routes,${ }^{34}$ but spatial resolutions were restricted to $\sim$ hundreds of nanometers. Evidently, it is crucial to characterize the spectral nature of such systems with high spatial and spectral resolutions, in order to understand the contributions of defects and impurities that can be as small a few nanometers in size. $^{35,36}$

The possibility of detecting optical forces using a scanning AFM tip has recently been utilized in the context of photoinduced force microscopy, ${ }^{37-39}$ to visualize the near-field optical response of polymers $^{39}$ and plasmonic structures ${ }^{40,41}$ with very high temporal ${ }^{42}$ and spatial resolutions down to $<10$ $\mathrm{nm}^{41,43}$. This idea behind using a multimodal approach to AFM-based imaging enables topological mapping and the ability to detect light-induced tip-sample forces simultaneously. The detected optical forces are shown to be sensitive to the local electric fields of materials, with extremely good polarization and spectral sensitivity. ${ }^{38,40}$ The spectral profile of the optical force is also expected to track the absorption spectrum in plasmonic structures. ${ }^{41,44}$

Here, we measure photoinduced forces in atomically thin layers of $\mathrm{MoS}_{2}$ and $\mathrm{WS}_{2}$ flakes on glass substrates using a modified commercial AFM. $\mathrm{MoS}_{2}$ and $\mathrm{WS}_{2}$ are both TMDs with a direct-to-indirect bandgap transition and characteristic excitonic peaks in the visible region of the optical spectrum. ${ }^{2,3} \mathrm{We}$ measure the near-field optical force spectra in the two materials and compare their excitonic signatures (in optical force) to the absorption measured by far-field spectroscopy. We also show that the force and correspondingly the absorption spectra, are strongly dependent on thicknesses and spatial heterogeneities in the TMD layers, by comparing two-dimensional force images acquired at several 
wavelengths. Finally, we show that the technique is particularly suited to image defects, grain boundaries and spatial inhomogeneities at very high spatio-spectral resolutions.

In our experiments, we utilized an in-vacuum AFM from Bruker, coupled to a home-built optoelectrical setup to be able to facilitate the synchronous imaging of topography and optical force (Fig. 1). A supercontinuum Fianium SC400 laser source and a SuperK VARIA wavelength filter with broadband tunability across the visible spectrum (NKT Photonics) provided the necessary optical excitation for force measurements. An electro-optic modulator (EOM) modulator was used to modulate the power of the laser at specific frequencies. The focal spot of the beam was controlled using a lens $(1.5 \mathrm{~cm}$ focal point), generating a spot with diameter of about 15 micrometers on the sample plane, resulting in an optical fluence of about $4.8 \mathrm{~mJ} / \mathrm{cm}^{2}$. The AFM cantilever and the sample were enclosed inside a custombuilt vacuum chamber attached to a vacuum pumping system, which provided us with the option of performing measurements under high vacuum $\left(<10^{-5} \mathrm{mbar}\right)$ for improved sensitivity.

The optical force and topographical information were acquired simultaneously in a bimodal fashion. ${ }^{42}$ This was achieved by modulating the incident laser power at frequency $f_{m}$, very close to the first order resonance frequency of the AFM cantilever $f_{0}$. The cantilever deflection was monitored using a quadrant photodetector as the tip is raster scanned along the sample. The cantilever deflection signal was then demodulated using two lock-in amplifiers, at frequencies $f_{m}$ and $f_{l}$ (the second order resonance frequency of the cantilever - used to detect topography), as shown in Fig. 1. We used silicon tips coated with Pt-Ir or Au with $f_{0}$ between 50 and $100 \mathrm{kHz}$ and $f_{l}$ between 300 and $700 \mathrm{kHz}$. MoS $\mathrm{S}_{2}$ and $\mathrm{WS}_{2}$ flakes were mechanically exfoliated and transferred onto separate glass slides of about 300 microns thick. For both samples, we identified regions consisting of mono- to few-layers of the respective TMD flakes. 
Fig. 2a shows the topography acquired on the $\mathrm{MoS}_{2}$ sample in our force microscopy setup, in a region consisting of two stacked $\mathrm{MoS}_{2}$ flakes (Supplemental Figure 1), acquired using a silicon tip coated with Pt-Ir. These measurements on the $\mathrm{MoS}_{2}$ sample were performed in air (vacuum chamber not pumped). The laser modulation frequency was set at $f_{m}=f_{0}+\Delta f=71 \mathrm{kHz}\left(f_{0}=70 \mathrm{kHz}\right)$ and the photoinduced force image, acquired simultaneously with the topographical image, is shown in Fig. $2 b$. The wavelength of the incident laser was chosen to be $\lambda=610 \mathrm{~nm}$, which is close to the expected ' $B$ ' exciton resonance in $\mathrm{MoS}_{2}$, as will be addressed further in the discussion below. It can be seen from the photoinduced force image that the contrast between the glass substrate and $\mathrm{MoS}_{2}$ is relatively good in comparison to topography. However, the force signal from $\mathrm{MoS}_{2}$ (at $f_{m}=71 \mathrm{kHz}$ ) is weaker relative to the glass substrate. This behavior can be understood as an interplay between the tunable modulation frequency of the incident beam $\left(f_{m}\right)$ and the natural resonance frequency of the cantilever $\left(f_{0}\right)$, as shown in Fig. 2c. The resonance frequency of the cantilever is affected by all optical forces exerted on the tip, including the gradient and scattering forces, ${ }^{37}$ (Citation here maybe), but also non-optical dispersive forces that strongly fall off away from the surface ${ }^{43}$. In our case, the cantilever resonance peak shifts to lower frequencies when the tip scans over $\mathrm{MoS}_{2}$, in comparison to when it is on glass as can be schematically seen by monitoring the demodulated amplitude of the cantilever at $f_{m}$ (Fig. 2c). This indicates that overall photoinduced force signal that is measured by the tip, is predominantly sensitive to the gradient component force (which is attractive in nature) and the contribution from scattering or dispersive forces is negligible, although present, in comparison. Indeed, as the measured photoinduced force signal is the amplitude determined by the lock-in at $f_{m}$, choosing the modulation frequency higher than the cantilever resonance peak $\left(f_{m}=f_{0}+\Delta f\right)$ results in an overall larger amplitude at $f_{m}$ on glass than on $\mathrm{MoS}_{2}$. Conversely, if $f_{m}=f_{0}-\Delta f$, then the force signal is expected to be stronger on $\mathrm{MoS}_{2}$, resulting in a reversal of contrast in the photoinduced force image. This behavior can be seen from the force image 
(Fig. 2e), when the incident beam is modulated at $f_{m}=69 \mathrm{kHz}$, while the contrast in topography is unaltered irrespective of $f_{m}$ (Fig. 2d). The cantilever resonance frequency, however, shifts in the same direction (towards lower frequencies on $\mathrm{MoS}_{2}$ ), irrespective of the modulation frequency $f_{m}$. Note that the relative contrast in the photoinduced force signal between the substrate and the surface of a material is also a function of the tip-sample distance. ${ }^{43}$ Hence, this technique is extremely sensitive to small variations in the dielectric environment of the surface that is probed by the tip. ${ }^{39,43}$ The sensitivity is stronger in vacuum, where the cantilever quality factors are higher and the resonances much sharper, as we discuss further.

Our setup enabled us to measure the photoinduced force as a function of the incident laser wavelength, across the visible region of the spectrum. Fig. 3a shows the photoinduced force spectrum acquired on the $\mathrm{MoS}_{2}$ sample, in the same region as that is shown in Fig. 2a. The force spectrum was obtained by scanning a $3 \mu \mathrm{m}$ line only along the horizontal axis (Y-scan axis disabled), with the first half of the line on $\mathrm{MoS}_{2}$, and the second half on glass As a result, the AFM acquisition software acquires a rectangular image as vertical stacks of the region along the same horizontal line. We simultaneously varied the wavelength of the incident laser, resulting in topography and photoinduced force images with the frequency (wavelength) and distance as the vertical and horizontal axes, respectively (Supplemental Figure 2). In order to obtain the photoinduced force spectrum purely from $\mathrm{MoS}_{2}$, we subtract the recovered spectrum obtained on glass from the values obtained over the region consisting of $\mathrm{MoS}_{2}$. Thus, we were able to eliminate any parasitic effects due to photo-thermal heating, background absorption, or laser power fluctuations, from the final retrieved spectrum shown in Fig. 3a. The evaluated photoinduced force spectrum (blue trace, Fig. 3a) is fit with a multi-Gaussian function, which reveals the presence of two-peaks in the plotted region, at $\sim 1.88 \mathrm{eV}(659 \mathrm{~nm})$ and $2.01 \mathrm{eV}(616 \mathrm{~nm})$, respectively. We compare this evaluated force spectrum to the absorption spectrum measured on a few- 
layer $\mathrm{MoS}_{2}$ sample deposited on glass using a far field UV-spectrophotometer (from Agilent) shown in Fig. $3 b$. The origin of the two excitonic resonances is due to the transition from the split valence band maxima to the conduction band minimum of the K-point of the Brillion zone in TMDs. ${ }^{2,4}$ The absorption spectrum confirms the presence of two distinct spectral features, which peak positions are in relatively good agreement with the measured photoinduced force spectrum.

Fig. 3c shows the photoinduced force spectrum of $\mathrm{WS}_{2}$ flakes, evaluated using the same procedure that was used for the $\mathrm{MoS}_{2}$ sample. However, all measurements on $\mathrm{WS}_{2}$ were performed in vacuum, while using a gold coated silicon tip. The optical absorption spectrum measured on the $\mathrm{WS}_{2}$ sample is shown in Fig. 3d. The experimental force signal (blue trace), fit with a multi-Gaussian function (black trace), confirms the presence of two excitonic resonance features at $2.02 \mathrm{eV}(613 \mathrm{~nm})$ and $2.33 \mathrm{eV}(532 \mathrm{~nm})$, once again in relatively good agreement with the optical absorption spectrum. The red-trace indicates the force spectrum acquired on a nearby neighboring region on the $\mathrm{WS}_{2}$ surface, indicating good reproducibility of our retrieval technique. Note that the sharp kinks in the force spectra of $\mathrm{MoS}_{2}$ and $\mathrm{WS}_{2}$ are only a consequence of our retrieval subtraction procedure. The far-field UV-vis absorption measurements on both $\mathrm{MoS}_{2}$ and $\mathrm{WS}_{2}$ flakes were measured on large areas of the sample (in relation to the sizes of individual flakes), consisting of domains ranging from mono- and few-layers to regions $>10 \mathrm{~nm}$ in thickness. Although the positions of $A$ and $B$ excitonic peaks are expected to be largely unaltered in few layers of TMDs in comparison to bulk structures (expected shifts only $\sim$ few tens of meV), ${ }^{5,33}$ the absorption at higher energies could very well be more sensitive to the sample thickness. This reasoning, in combination with the fact that the overall absorption approaching UV frequencies depends strongly on the absorption of the dielectric substrate, could rationalize the discrepancy between absorption and force spectra $(>2.5 \mathrm{eV})$ in our experiments. Nevertheless, the ability to obtain spectral information (in the visible domain) in atomically thin structures with high 
spectral and spatial resolutions, adds a new dimension to nanoscale imaging in the context of photoinduced force microscopy.

Fig. 4a shows the topography acquired on a region consisting of $\mathrm{WS}_{2}$ flakes on glass with evident non-uniformity in the flake thickness, indicating the presence of additional layers. The corresponding photoinduced force image acquired at $2.28 \mathrm{eV}(\lambda=545 \mathrm{~nm})$ is shown in Fig. $4 \mathrm{~b}$. The variation of the photoinduced force signal on the imaged $\mathrm{WS}_{2}$ flakes, is an evidence of the thicknessdependence of absorption manifesting, as enhanced contrast between different regions on $\mathrm{WS}_{2}$. The force image most notably reveals regions of overlapping flakes and defects that are not resolved in topography image. To further elucidate the site-dependent nature of absorption, we recorded the photoinduced force spectrum on two separate regions on $\mathrm{WS}_{2}$, indicated by the red (region 1) and blue squares (region 2) in the topography image (Fig. 4a), as shown in Fig. 4c. The red and blue regions correspond to regions with varying thicknesses $-1.7 \mathrm{~nm}(\sim 2$ layers $)$ and $3.6 \mathrm{~nm}(\sim 5$ layers $)$, respectively (Supplemental Figure 3). The corresponding force spectra (Fig. 4c) clearly reveal the thickness-dependence of the $A$ and $B$ excitonic peaks, as also reported by hyperspectral microscopy studies of absorption on 2D structures. ${ }^{33}$ Similarly, site-dependent photoluminescence studies have exploited the property of direct-to-indirect bandgap transition in TMDs to distinguish monolayers from thicker regions.

However, the superior spatial resolution achieved in our technique and the access to highly local, near-field information, enables the possibility of characterization of defects, imperfections and grain boundaries in 2D materials spatially and spectroscopically. We present an initial proof-of-concept demonstration of this idea in Fig. 4. Fig. 4d shows the force images acquired at three different wavelengths on a region of $\mathrm{WS}_{2}$ as depicted (by the white rectangle) in the topography image in Fig. 4a. It is evident from the 2D maps of photoinduced force profiles that the boundary between two conjoined 
flakes (indicated by the dotted square in the top panel, Fig. 4d) is best resolved at $1.92 \mathrm{eV}(646 \mathrm{~nm})$, compared to the other two wavelengths. Similarly, defects on the surface and on the edge of a multilayer flake (indicated by the dotted squares in the middle panel) are most clearly resolved at $2.28 \mathrm{eV}(\sim 544$ $\mathrm{nm})$. However, the same surface and its defects are almost entirely unresolved at $2.72 \mathrm{eV}(456 \mathrm{~nm}) \mathrm{in}$ the force image (bottom panel, Fig. 4d)

In summary, we demonstrated a way to elicit the morphological and photoinduced force profiles in 2D materials $\left(\mathrm{MoS}_{2}\right.$ and $\left.\mathrm{WS}_{2}\right)$ in a synchronous fashion, using an AFM force-based near-field imaging technique. We also evaluated the spectral nature of photoinduced forces in atomically thin layers of both the 2D materials separately. Our spectral force retrieval technique enables us to evaluate the distinct excitonic peaks of the two structures in the visible range, which qualitatively resemble the excitonic features of far-field absorption. We infer that the strength and position of excitonic peaks in the force spectra of 2D layers are sensitive to the sample thickness, which opens the door towards segregating the spectral response of single layers of atomically thin 2D structures from multilayered stacks, including in complex heterostructures. We also observed spectral signatures of strong contrast differences between defects, edges and the surface of $\mathrm{WS}_{2}$ flakes, potentially paving the way towards non-destructive defect imaging of 2D materials. 


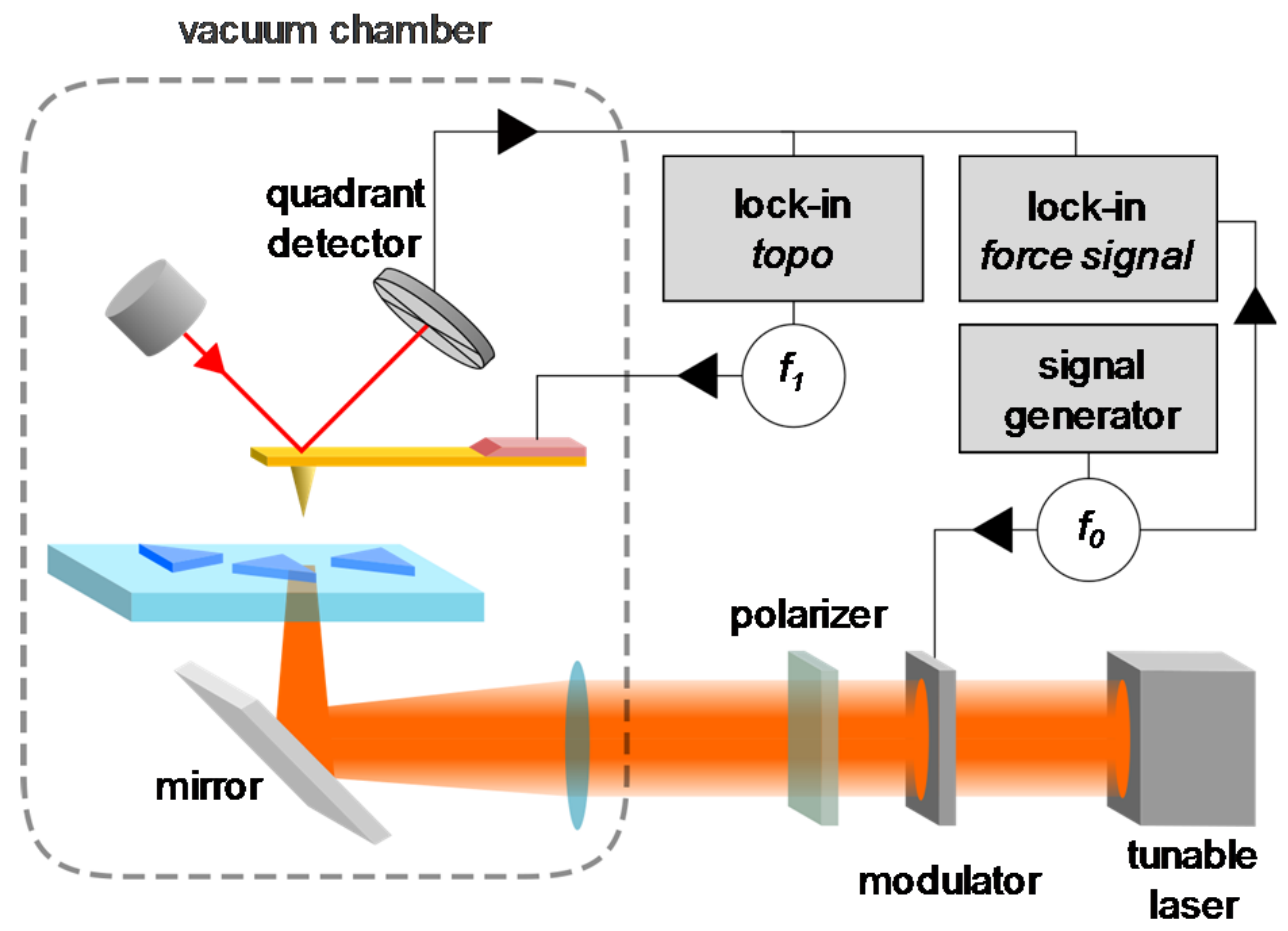

Fig. 1. Schematic of the AFM-based setup used for photoinduced force imaging. 

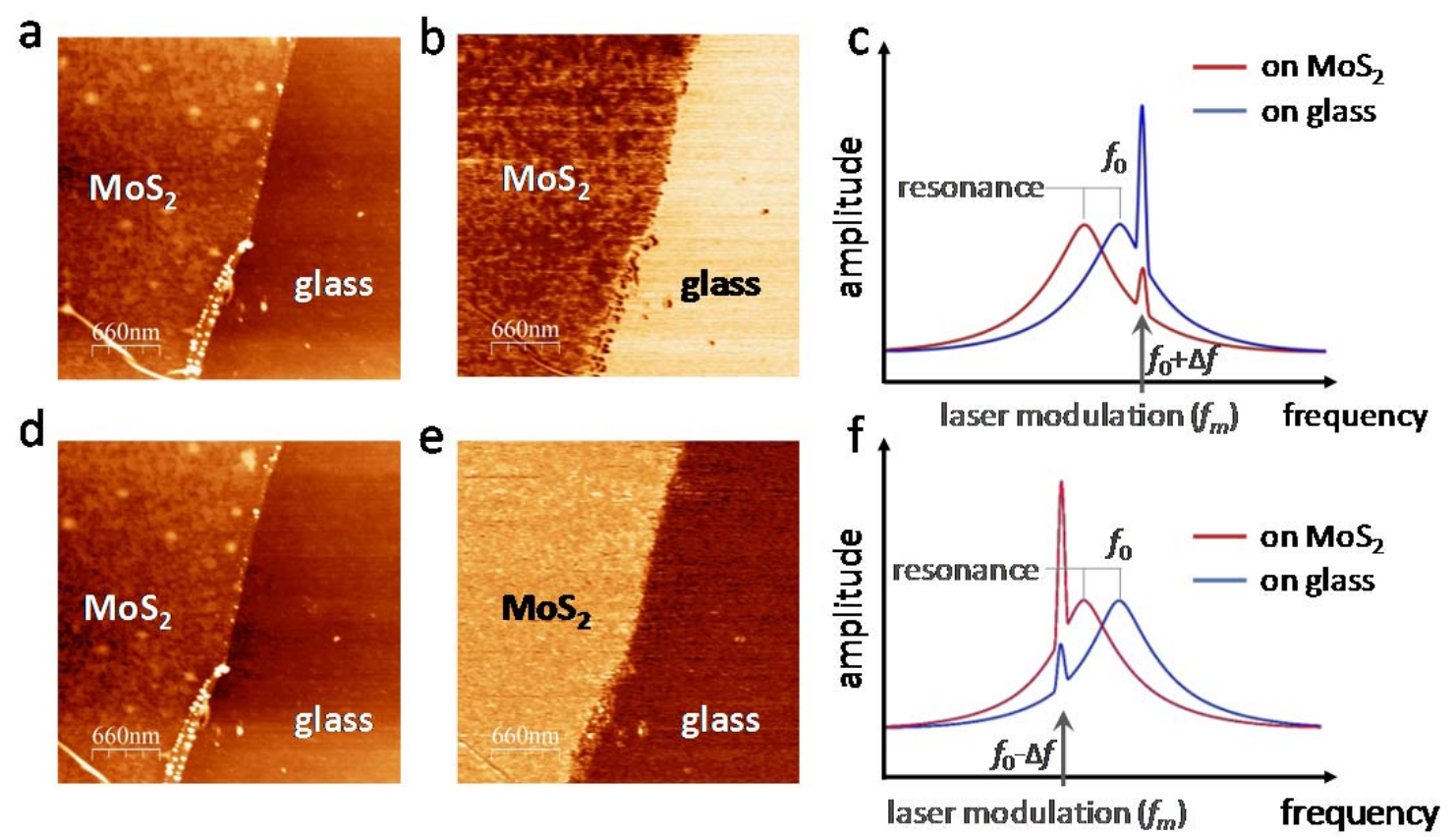

Fig. 2. (a), (b) The topography and photoinduced force maps of a region consisting of two $\mathrm{MoS}_{2}$ flakes on glass, acquired by modulating the laser at $f_{m}=71 \mathrm{kHz}$. (c) A representative schematic of the lock-in amplitude as a function of the lock-in detuning frequency when the tip is positioned on top of glass (blue trace) and on top of $\mathrm{MoS}_{2}$ (red trace). Similarly, (d) and (e) show the topography and photoinduced force maps acquired at $f_{m}=69 \mathrm{kHz}$. (f) same as panel (c), for the case when $f_{m}<f_{0}$. 

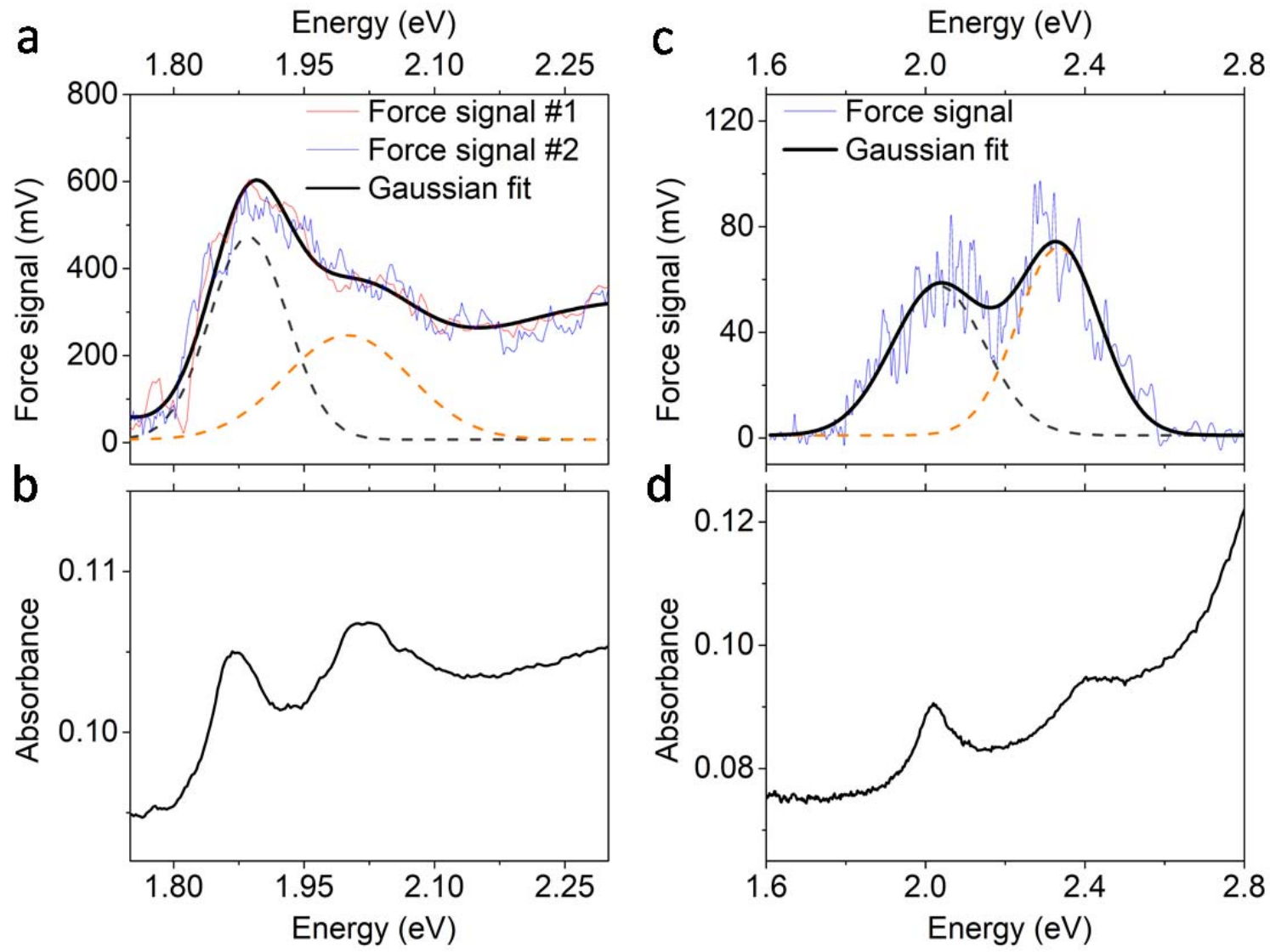

Fig. 3. Photoinduced force spectra acquired on a sample consisting of (a) $\mathrm{MoS}_{2}$ and (c) $\mathrm{WS}_{2}$ flakes deposited on separate glass substrates. The blue traces are the experimental force signals, retrieved as a function of wavelength. The solid black lines are the Gaussian fits of the measured force spectra and the dashed lines indicate the residual peaks of the corresponding cumulative Gaussian function. The red trace in panel (a) indicates the force spectrum retrieved close to the spot where the blue trace was acquired. (b) and (d) Far-field absorption spectra obtained on the $\mathrm{MoS}_{2}$ and $\mathrm{WS}_{2}$ samples, respectively. 

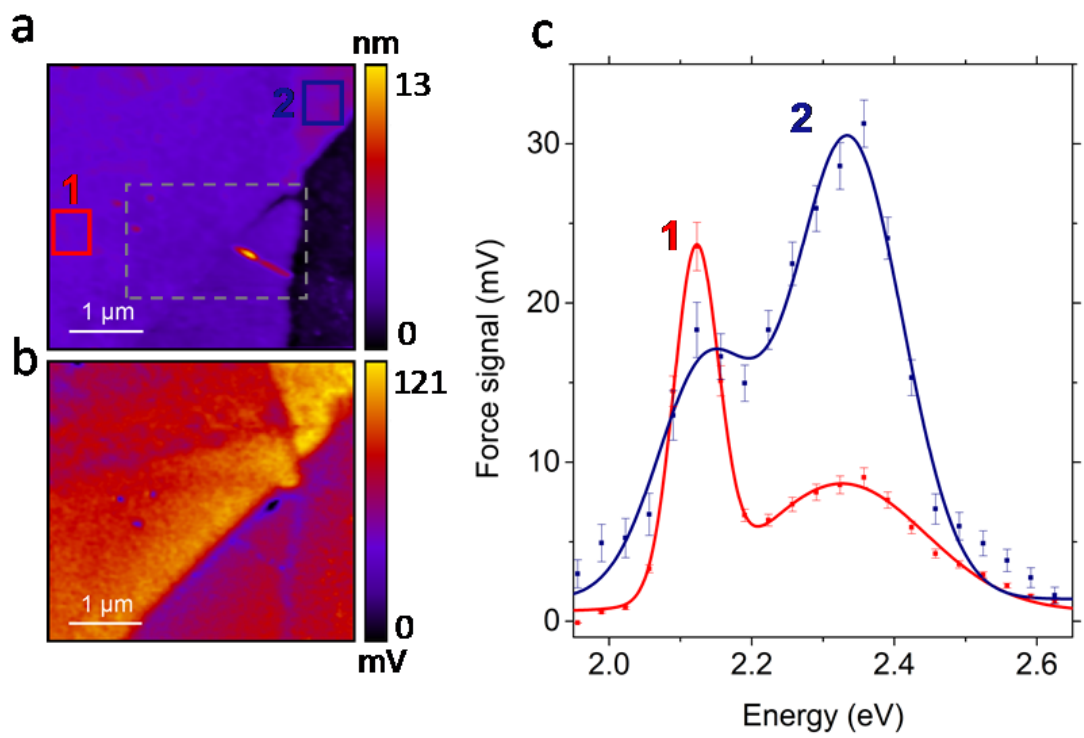

d
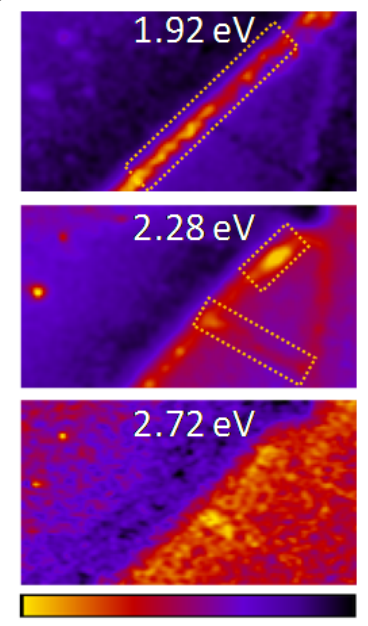

Fig. 4. (a) Topography and (b) a representative photoinduced force map acquired on the $\mathrm{WS}_{2}$ sample (at $2.28 \mathrm{eV}$ or $\lambda=545 \mathrm{~nm}$ ), indicating regions with varying thicknesses and flake orientations on the neighboring glass substrate. (c) Photoinduced force spectra averaged over regions 1 (red trace) and 2(blue trace), as indicated by the squares in panel (a). (d) Photoinduced force maps acquired at three different wavelengths over the same region as that indicated by the dashed rectangle in panel (a). The dotted yellow rectangles in panel (d) indicate the boundary between two flakes (top panel), or defects on the surface or the edges of one multilayer flake (middle panel).

Acknowledgements: We thank N. Beyer for technical assistance. ANR OH-RISQUE SMARAGD (14 OHRI 0008 01), EOARD (FA8655-13-1-3001) and partially by ANR-11-LABX-0058-NIE within the Investissement d'Avenir program ANR-10-IDEX-0002-02. 


\section{References:}

${ }^{1}$ K.S. Novoselov, D. Jiang, F. Schedin, T.J. Booth, V. V Khotkevich, S. V Morozov, and A.K. Geim, Proc. Natl. Acad. Sci. U. S. A. 102, 10451 (2005).

${ }^{2}$ Q.H. Wang, K. Kalantar-Zadeh, A. Kis, J.N. Coleman, and M.S. Strano, Nat. Nanotechnol. 7, 699 (2012).

${ }^{3}$ K.F. Mak and J. Shan, Nat. Photonics 10, 216 (2016).

${ }^{4}$ K.F. Mak, C. Lee, J. Hone, J. Shan, and T.F. Heinz, Phys. Rev. Lett. 105, 136805 (2010).

${ }^{5}$ Y. Li, A. Chernikov, X. Zhang, A. Rigosi, H.M. Hill, A.M. Van Der Zande, D.A. Chenet, E.M. Shih, J. Hone, and T.F. Heinz, Phys. Rev. B - Condens. Matter Mater. Phys. 90, 1 (2014).

${ }^{6}$ A. Splendiani, L. Sun, Y. Zhang, T. Li, J. Kim, C.-Y. Chim, G. Galli, and F. Wang, Nano Lett. 10, 1271 (2010).

${ }^{7}$ T. Korn, S. Heydrich, M. Hirmer, J. Schmutzler, and C. Schüller, Appl. Phys. Lett. 99, 102109 (2011).

${ }^{8}$ M. Palummo, M. Bernardi, and J.C. Grossman, Nano Lett. 15, 2794 (2015).

${ }^{9}$ T. Cheiwchanchamnangij and W.R.L. Lambrecht, Phys. Rev. B 85, 205302 (2012).

${ }^{10}$ A. Chernikov, T.C. Berkelbach, H.M. Hill, A. Rigosi, Y. Li, O.B. Aslan, D.R. Reichman, M.S. Hybertsen, and T.F. Heinz, Phys. Rev. Lett. 113, 076802 (2014).

${ }^{11}$ A.T. Hanbicki, M. Currie, G. Kioseoglou, A.L. Friedman, and B.T. Jonker, Solid State Commun. 203, $16(2015)$

${ }^{12}$ Q. Lu, Y. Yu, Q. Ma, B. Chen, and H. Zhang, Adv. Mater. 28, 1917 (2016).

${ }^{13}$ W. Peng, Y. Li, F. Zhang, G. Zhang, and X. Fan, Ind. Eng. Chem. Res. 56, 4611 (2017).

${ }^{14}$ X. Liu, T. Galfsky, Z. Sun, F. Xia, E. Lin, Y.-H. Lee, S. Kéna-Cohen, and V.M. Menon, Nat. Photonics 9, 30 (2015).

${ }^{15}$ S. Wang, S. Li, T. Chervy, A. Shalabney, S. Azzini, E. Orgiu, J.A. Hutchison, C. Genet, P. Samorì, and T.W. Ebbesen, Nano Lett. 16, 4368 (2016).

${ }^{16}$ Y. Kang, S. Najmaei, Z. Liu, Y. Bao, Y. Wang, X. Zhu, N.J. Halas, P. Nordlander, P.M. Ajayan, J. Lou, and Z. Fang, Adv. Mater. 26, 6467 (2014).

${ }^{17}$ X. Gan, Y. Gao, K. Fai Mak, X. Yao, R.-J. Shiue, A. van der Zande, M.E. Trusheim, F. Hatami, T.F. Heinz, J. Hone, and D. Englund, Appl. Phys. Lett. 103, 181119 (2013).

${ }^{18}$ J. Kern, A. Trügler, I. Niehues, J. Ewering, R. Schmidt, R. Schneider, S. Najmaei, A. George, J. Zhang, J. Lou, U. Hohenester, S. Michaelis de Vasconcellos, and R. Bratschitsch, ACS Photonics 2, 1260 (2015).

${ }^{19}$ S.S. Singha, D. Nandi, and A. Singha, RSC Adv. 5, 24188 (2015). 
${ }^{20}$ Y. Ye, Z.J. Wong, X. Lu, X. Ni, H. Zhu, X. Chen, Y. Wang, and X. Zhang, Nat. Photonics 9, 733 (2015).

${ }^{21}$ S. Wu, S. Buckley, J.R. Schaibley, L. Feng, J. Yan, D.G. Mandrus, F. Hatami, W. Yao, J. Vučković, A. Majumdar, and X. Xu, Nature 520, 69 (2015).

${ }^{22}$ H. Nan, Z. Wang, W. Wang, Z. Liang, Y. Lu, Q. Chen, D. He, P. Tan, F. Miao, X. Wang, J. Wang, and Z. Ni, ACS Nano 8, 5738 (2014).

${ }^{23}$ M. Amani, D. Lien, D. Kiriya, J. Xiao, A. Azcatl, J. Noh, S.R. Madhvapathy, R. Addou, S. Kc, M. Dubey, K. Cho, R.M. Wallace, S. Lee, J. He, J.W.A. Iii, X. Zhang, E. Yablonovitch, and A. Javey, Science 350, 1065 (2015).

${ }^{24}$ W. Zhou, X. Zou, S. Najmaei, Z. Liu, Y. Shi, J. Kong, J. Lou, P.M. Ajayan, B.I. Yakobson, and J.-C. Idrobo, Nano Lett. 13, 2615 (2013).

${ }^{25}$ M. Ghorbani-Asl, A.N. Enyashin, A. Kuc, G. Seifert, and T. Heine, Phys. Rev. B 88, 245440 (2013).

${ }^{26}$ S. Mouri, Y. Miyauchi, M. Toh, W. Zhao, G. Eda, and K. Matsuda, Phys. Rev. B 90, 155449 (2014).

${ }^{27}$ X. Yin, Z. Ye, D.A. Chenet, Y. Ye, K. O’Brien, J.C. Hone, and X. Zhang, Science 344, 488 (2014).

${ }^{28}$ Z. Wu and Z. Ni, Nanophotonics 6, 1219 (2017).

${ }^{29}$ W. Bao, N.J. Borys, C. Ko, J. Suh, W. Fan, A. Thron, Y. Zhang, A. Buyanin, J. Zhang, S. Cabrini, P.D. Ashby, A. Weber-Bargioni, S. Tongay, S. Aloni, D.F. Ogletree, J. Wu, M.B. Salmeron, and P.J. Schuck, Nat. Commun. 6, 7993 (2015).

${ }^{30}$ Y. Lee, S. Park, H. Kim, G.H. Han, Y.H. Lee, and J. Kim, Nanoscale 7, 11909 (2015).

${ }^{31}$ K.-D. Park, O. Khatib, V. Kravtsov, G. Clark, X. Xu, and M.B. Raschke, ArXiv 1601.01364 (2016).

32 J. Nozaki, S. Mori, Y. Miyata, Y. Maniwa, and K. Yanagi, Jpn. J. Appl. Phys. 55, 3 (2016).

${ }^{33}$ A. Castellanos-Gomez, J. Quereda, H.P. van der Meulen, N. Agrait, and G. Rubio-Bollinger, Nanotechnology 27, 5 (2016).

${ }^{34}$ K.P. Dhakal, D.L. Duong, J. Lee, H. Nam, M. Kim, M. Kan, Y.H. Lee, and J. Kim, Nanoscale 6, 13028 (2014).

${ }^{35}$ H. Liu, H. Zheng, F. Yang, L. Jiao, J. Chen, W. Ho, C. Gao, J. Jia, and M. Xie, ACS Nano 9, 6619 (2015).

${ }^{36}$ P. Vancsó, G.Z. Magda, J. Pető, J.-Y. Noh, Y.-S. Kim, C. Hwang, L.P. Biró, and L. Tapasztó, Sci. Rep. 6, 29726 (2016).

${ }^{37}$ J. Jahng, J. Brocious, D. a. Fishman, F. Huang, X. Li, V.A. Tamma, H.K. Wickramasinghe, and E.O. Potma, Phys. Rev. B 90, 155417 (2014).

${ }^{38}$ F. Huang, V.A. Tamma, Z. Mardy, J. Burdett, and H.K. Wickramasinghe, Sci. Rep. 5, 10610 (2015).

${ }^{39}$ D. Nowak, W. Morrison, H.K. Wickramasinghe, J. Jahng, E. Potma, L. Wan, R. Ruiz, T.R. Albrecht, 
K. Schmidt, J. Frommer, D.P. Sanders, and S. Park, Sci. Adv. 2, e1501571 (2016).

${ }^{40}$ T.U. Tumkur, X. Yang, B. Cerjan, N.J. Halas, P. Nordlander, and I. Thomann, Nano Lett. 16, 7942 (2016).

${ }^{41}$ T. Tumkur, X. Yang, C. Zhang, J. Yang, Y. Zhang, G.V. Naik, P. Nordlander, and N.J. Halas, Nano Lett. 18, (2018).

${ }^{42}$ J. Jahng, D.A. Fishman, S. Park, D.B. Nowak, W.A. Morrison, H.K. Wickramasinghe, and E.O. Potma, Acc. Chem. Res. 48, 2671 (2015).

${ }^{43}$ A. Ambrosio, R.C. Devlin, F. Capasso, and W.L. Wilson, ACS Photonics 4, 846 (2017).

${ }^{44}$ H.U. Yang and M.B. Raschke, New J. Phys. 18, 1 (2016). 